\title{
Stochastic orderings with respect to a capacity and an application to a financial optimization problem
}

\author{
Miryana Grigorova \\ LPMA, Université Paris 7 \\ First version: July 4, 2010 \\ Present version: April 30, 2011
}

\begin{abstract}
In an analogous way to the classical case of a probability measure, we extend the notion of an increasing convex (concave) stochastic dominance relation to the case of a normalised monotone (but not necessarily additive) set function also called a capacity. We give different characterizations of this relation establishing a link to the notions of distribution function and quantile function with respect to the given capacity. The Choquet integral is extensively used as a tool. We state a new version of the classical upper (resp. lower) Hardy-Littlewood's inequality generalized to the case of a continuous from below concave (resp. convex) capacity. We apply our results to a financial optimization problem whose constraints are expressed by means of the increasing convex stochastic dominance relation with respect to a capacity.

Keywords: stochastic orderings, increasing convex stochastic dominance, Choquet integral, quantile function with respect to a capacity, stop-loss ordering, Choquet expected utility, distorted capacity, generalized Hardy-Littlewood's inequalities, distortion risk measure, ambiguity
\end{abstract}

The author is deeply grateful to Marie-Claire Quenez as well as to two anonymous referees and an associate editor for their helpful suggestions and remarks. 


\section{Introduction}

Capacities and integration with respect to capacities were introduced by Choquet and were afterwards applied in different areas such as economics and finance among many others (cf. for instance Wang and Yan 2007 for an overview of applications). In economics and finance, capacities and Choquet integrals have been used, in particular, to build alternative theories to the "classical" setting of expected utility maximization of Von Neumann and Morgenstern. Indeed, the classical expected utility paradigm has been challenged by various empirical experiments and "paradoxes" (such as Allais's and Ellsberg's) thus leading to the development of new theories. One of the proposed new paradigms is the Choquet expected utility (abridged as CEU) where agent's preferences are represented by a capacity $\mu$ and a non-decreasing real-valued function $u$. The agent's "satisfaction" with a claim $X$ is therefore assessed by the Choquet integral of $u(X)$ with respect to the capacity $\mu$. Choquet expected utility intervenes in situations where an objective probability measure is not given and where the agents are not able to derive a subjective probability over the set of different scenarios.

On the other hand, stochastic orders have also been extensively used in the decision theory. They represent partial order relations on the space of random variables on some probability space $(\Omega, \mathcal{F}, \mathbb{P})$ (more precisely, stochastic orders are partial order relations on the set of the corresponding distribution functions). Different kinds of orders have been studied and applied (see for instance Müller and Stoyan 2002 and Shaked and Shanthikumar 2006 for a general presentation) and links to the expected utility theory have been exploited. Hereafter, we will call "classical" the results on risk orders in the case of random variables on a probability space. In the classical setting of random variables on a probability space, there are two approaches to risk orderings - economic ordering based on classes of utility functions and statistical ordering which is based on tail distributions (cf. the explanations in Wang and Young 1998). In the "classical" case of a probability space they lead to the same ordering of risks. For the purpose of this paper we will focus on the increasing convex ordering (or increasing convex stochastic dominance relation). The economic approach to the classical increasing convex stochastic dominance amounts to the following definition - $X$ is said to be dominated by $Y$ in the increasing convex stochastic dominance relation (denoted $\left.X \leq_{i c x} Y\right)$ if $\mathbb{E}(u(X)) \leq \mathbb{E}(u(Y))$ for all $u: \mathbb{R} \rightarrow \mathbb{R}$ non-decreasing and convex provided the expectations (taken in the Lebesgue sense) exist in $\mathbb{R}$. The economic 
interpretation is then the following: $X$ is dominated by $Y$ in the increasing convex stochastic dominance if $Y$ is preferred to $X$ by all decision makers who prefer more wealth to less and who are risk-seeking. The statistical approach provides an equivalent characterization: $X \leq_{i c x} Y$ if and only if $\int_{x}^{+\infty} \mathbb{P}(X>u) d u \leq \int_{x}^{+\infty} \mathbb{P}(Y>u) d u, \forall x \in \mathbb{R}$, provided the integrals exist in $\mathbb{R}$. Moreover, we have another characterization which establishes a link between the $i c x$ ordering relation and stop-loss premia in reinsurance (cf. Dhaene et al. 2006): $X \leq_{i c x} Y$ if and only if $\mathbb{E}\left((X-b)^{+}\right) \leq \mathbb{E}\left((Y-b)^{+}\right), \forall b \in \mathbb{R}$, provided the expectations exist in $\mathbb{R}$.

The first aim of this paper is to generalize the notion of increasing convex stochastic dominance to the case where the measurable space $(\Omega, \mathcal{F})$ is endowed with a given capacity $\mu$ which is not necessarily a probability measure and to investigate generalizations of the previously mentioned results to this setting. Of course, in our case "ordinary" expectations (in the Lebesgue sense) have to be replaced by Choquet expectations. We obtain that analogous kinds of characterizations as the previously mentioned in the case of a probability measure remain valid in our more general setting if we assume that the capacity $\mu$ has certain continuity properties (namely continuity from below and continuity from above). Nevertheless, let us remark that in all proofs but one the assumption of continuity from below and from above is not needed.

The second aim of this paper is to give an application of the results we obtain to a financial problem inspired by Dana (2005) (see also Dana and Meilijson 2003 and the references therein). The authors study the following problem:

$$
\text { Minimize } \mathbb{E}(Z C)
$$

under the constraints: $C \in L^{\infty}(\Omega, \mathcal{F}, \mathbb{P})$ such that $X \leq_{i c v} C$

where the abbreviation $i c v$ stands for the increasing concave ordering relation (with respect to the probability measure $\mathbb{P}$ ), the symbol $\mathbb{E}$ denotes Lebesgue expectation (with respect to the probability measure $\mathbb{P}$ ) and where $Z$ and $X$ are given (see below for more details). Let us just recall that the increasing concave stochastic dominance relation is defined similarly to the increasing convex stochastic dominance, the class of non-decreasing convex real-valued functions in the definition being replaced by the class of non-decreasing concave real-valued functions. The authors interpret the value function of the above problem $(\tilde{D})$ as being the minimal expenditure to get a contingent claim among those which dominate the contingent claim $X$ in the increasing concave ordering. The value function of the problem is linked to the notion of risk measure as well. By analogy with this problem we are interested in the following optimization problem 
where we are given a (continuous from below concave) capacity $\mu$ and a non-negative numéraire $Z$ :

$$
\underset{C \in \mathcal{A}(X)}{\operatorname{Maximize}} \mathbb{E}_{\mu}(Z C)
$$

the symbol $\mathbb{E}_{\mu}$ denoting the Choquet integral with respect to $\mu$ and $\mathcal{A}(X)$ standing for the set of all non-negative, bounded contingent claims $C$ which precede a given non-negative bounded contingent claim $X$ in the increasing convex ordering with respect to the capacity $\mu$ (cf. hereafter for a precise formulation of the problem). We can interpret the functional $C \mapsto \mathbb{E}_{\mu}(Z C)$ as a pricing functional, the measurable function $Z$ being interpreted as a discount factor; in fact, the idea of using Choquet integrals as non-linear pricing functionals in insurance and finance is not new (cf. for instance the overview in Wang and Yan 2007 and the references therein). The functional $\mathbb{E}_{\mu}($.$) can be seen as a risk measure as well (see below for more details); non-linear$ pricing functionals and risk measures have already been connected in the work of Bion-Nadal (2009) and Klöppel and Schweizer (2007).

We also give an interpretation of the value function of our problem in terms of a class of risk measures which we call "generalized" distortion risk measures (as well as in terms of premium principles in insurance). In order to solve problem (D), we state a new version of the classical Hardy-Littlewood's upper (resp. lower) inequality generalized to the case of a continuous from below concave (resp. convex) capacity. Then, using this result, we compute the value function of problem (D).

The rest of this paper is organized as follows. In section 2 we fix the terminology and the notation by giving some well-known definitions about capacities and Choquet integrals; in particular, the notions of comonotonic measurable functions and quantile function with respect to a capacity are recalled. In section 3 we define the notion of increasing convex (concave) stochastic dominance with respect to a capacity and explore different characterizations analogous to those existing in the classical case of a probability measure. In section 4 we state the "generalized" HardyLittlewood's inequalities. In section 5 we formulate and solve our optimization problem (D); in two subsections we provide an interpretation of the value function in terms of risk measures in finance and premium principles in insurance. Finally, in section 6 we present directions for our ongoing and future research concerning some related problems. The Appendix contains three parts: some well-known results about Choquet integration which are used in the paper 
are recalled in Appendix A; Appendix B is devoted to the proofs of a lemma and a proposition from section 3; the proof of the "generalized" Hardy-Littlewood's inequalities is presented in Appendix C.

\section{Notation and definitions}

The definitions and results of this section can be found in the book by Föllmer and Schied (2004) (cf. section 4.7 of this reference) and/or in the one by Denneberg (1994).

Let $(\Omega, \mathcal{F})$ be a measurable space. We denote by $\chi$ the space of measurable, real-valued and bounded functions on $(\Omega, \mathcal{F})$.

Definition 2.1 Let $(\Omega, \mathcal{F})$ be a measurable space. A set function $\mu: \mathcal{F} \longrightarrow[0,1]$ is called a capacity if it satisfies $\mu(\varnothing)=0, \mu(\Omega)=1$ (normalisation) and the following monotonicity property: $A, B \in \mathcal{F}, A \subset B \Rightarrow \mu(A) \leq \mu(B)$.

We recall the definition of the Choquet integral with respect to a capacity $\mu$ (cf. Denneberg 1994).

Definition 2.2 For a measurable real-valued function $X$ on $(\Omega, \mathcal{F})$, the Choquet integral with respect to a capacity $\mu$ is defined as follows

$$
\mathbb{E}_{\mu}(X):=\int_{0}^{+\infty} \mu(X>x) d x+\int_{-\infty}^{0}(\mu(X>x)-1) d x
$$

Note that the Choquet integral in the preceding definition may not exist (namely if one of the two (Riemann) integrals on the right side is equal to $+\infty$ and the other to $-\infty$ ), may be in $\mathbb{R}$ or may be equal to $+\infty$ or $-\infty$. The Choquet integral always exists if the function $X$ is bounded from below or from above. The Choquet integral exists and is finite if $X$ is in $\chi$.

Thus we come to the notion of the (non-decreasing) distribution function of $X$ with respect to a capacity $\mu$.

Definition 2.3 Let $X$ be a measurable function defined on $(\Omega, \mathcal{F})$. We call a distribution function of $X$ with respect to $\mu$ the non-decreasing function $G_{X}$ defined by

$$
G_{X}(x):=1-\mu(X>x), \quad \forall x \in \mathbb{R} .
$$


The non-decreasingness of $G_{X}$ in definition 2.3 is due to the monotonicity of $\mu$.

In the case where $\mu$ is a probability measure, the distribution function $G_{X}$ coincides with the usual distribution function $F_{X}$ of $X$ defined by $F_{X}(x):=\mu(X \leq x), \quad \forall x \in \mathbb{R}$.

Let us now define the generalized inverse of the function $G_{X}$.

Definition 2.4 For a measurable real-valued function $X$ defined on $(\Omega, \mathcal{F})$ and for a capacity $\mu$, let $G_{X}$ denote the distribution function of $X$ with respect to $\mu$. We call a quantile function of $X$ with respect to $\mu$ every function $r_{X}:(0,1) \longrightarrow \overline{\mathbb{R}}$ verifying

$$
\sup \left\{x \in \mathbb{R} \mid G_{X}(x)<t\right\} \leq r_{X}(t) \leq \sup \left\{x \in \mathbb{R} \mid G_{X}(x) \leq t\right\}, \forall t \in(0,1)
$$

where the convention $\sup \{\varnothing\}=-\infty$ is used.

The functions $r_{X}^{l}$ and $r_{X}^{u}$ defined by

$r_{X}^{l}(t):=\sup \left\{x \in \mathbb{R} \mid G_{X}(x)<t\right\}, \forall t \in(0,1)$ and $r_{X}^{u}(t):=\sup \left\{x \in \mathbb{R} \mid G_{X}(x) \leq t\right\}, \forall t \in(0,1)$

are called the lower and upper quantile functions of $X$ with respect to $\mu$.

For notational convenience, we omit the dependence on $\mu$ in the notation $G_{X}$ and $r_{X}$ when there is no ambiguity.

Remark 2.1 Let $\mu$ be a capacity and let $X$ be a measurable real-valued function such that

$$
\lim _{x \rightarrow-\infty} G_{X}(x)=0 \text { and } \lim _{x \rightarrow+\infty} G_{X}(x)=1 .
$$

We denote by $G_{X}(x-)$ and $G_{X}(x+)$ the left-hand and right-hand limits of $G_{X}$ at $x$. A function $r_{X}$ is a quantile function of $X$ (with respect to $\mu$ ) if and only if

$$
\left(G_{X}\left(r_{X}(t)-\right) \leq t \leq G_{X}\left(r_{X}(t)+\right), \quad \forall t \in(0,1)\right.
$$

In this case $r_{X}$ is real-valued. Note that the condition (2.1) is satisfied if $X \in \chi$ and $\mu$ is arbitrary. The condition (2.1) is satisfied for an arbitrary $X$ if $\mu$ is continuous from below and from above (see definition 2.5).

We recall some well-known definitions about capacities which will be needed later on.

Definition 2.5 A capacity $\mu$ is called convex (or equivalently, supermodular) if

$$
A, B \in \mathcal{F} \Rightarrow \mu(A \cup B)+\mu(A \cap B) \geq \mu(A)+\mu(B) .
$$


A capacity $\mu$ is called concave (or submodular, or 2-alternating) if

$$
A, B \in \mathcal{F} \Rightarrow \mu(A \cup B)+\mu(A \cap B) \leq \mu(A)+\mu(B) .
$$

A capacity $\mu$ is called continuous from below if

$$
\left(A_{n}\right) \subset \mathcal{F} \text { such that } A_{n} \subset A_{n+1}, \forall n \in \mathbb{N} \Rightarrow \lim _{n \rightarrow \infty} \mu\left(A_{n}\right)=\mu\left(\cup_{n=1}^{\infty} A_{n}\right) .
$$

A capacity $\mu$ is called continuous from above if

$$
\left(A_{n}\right) \subset \mathcal{F} \text { such that } A_{n} \supset A_{n+1}, \forall n \in \mathbb{N} \Rightarrow \lim _{n \rightarrow \infty} \mu\left(A_{n}\right)=\mu\left(\cap_{n=1}^{\infty} A_{n}\right) .
$$

We recall the notion of comonotonic functions (cf. Föllmer and Schied 2004).

Definition 2.6 Two measurable functions $X$ and $Y$ on $(\Omega, \mathcal{F})$ are called comonotonic if

$$
\left(X(\omega)-X\left(\omega^{\prime}\right)\right)\left(Y(\omega)-Y\left(\omega^{\prime}\right)\right) \geq 0, \forall\left(\omega, \omega^{\prime}\right) \in \Omega \times \Omega .
$$

For reader's convenience and in order to fix the terminology, we summarize some of the main properties of Choquet integrals in the following propositions (cf. proposition 5.1 in Denneberg 1994) and we make the convention that the properties are valid provided the expressions make sense (which is always the case when we restrain ourselves to elements in $\chi$ ).

Proposition 2.1 Let $\mu$ be a capacity on $(\Omega, \mathcal{F})$ and $X$ and $Y$ be measurable real-valued functions on $(\Omega, \mathcal{F})$, then we have the properties:

- (positive homogeneity) $\mathbb{E}_{\mu}(\lambda X)=\lambda \mathbb{E}_{\mu}(X), \forall \lambda \in \mathbb{R}_{+}$

- (monotonicity) $X \leq Y \Rightarrow \mathbb{E}_{\mu}(X) \leq \mathbb{E}_{\mu}(Y)$

- (translation invariance) $\mathbb{E}_{\mu}(X+b)=\mathbb{E}_{\mu}(X)+b, \forall b \in \mathbb{R}$

- (asymmetry) $\mathbb{E}_{\mu}(-X)=-\mathbb{E}_{\bar{\mu}}(X)$, where $\bar{\mu}$ is the dual capacity of $\mu$ $\left(\bar{\mu}(A)\right.$ is defined by $\left.\bar{\mu}(A)=1-\mu\left(A^{c}\right), \forall A \in \mathcal{F}\right)$

- (comonotonic additivity) If $X$ and $Y$ are comonotonic, then $\mathbb{E}_{\mu}(X+Y)=\mathbb{E}_{\mu}(X)+\mathbb{E}_{\mu}(Y)$.

Finally, we remind the subadditivity property of the Choquet integral with respect to a concave capacity. 
Proposition 2.2 Let $\mu$ be a concave capacity on $(\Omega, \mathcal{F})$ and $X$ and $Y$ be measurable real-valued functions on $(\Omega, \mathcal{F})$ such that $\mathbb{E}_{\mu}(X)>-\infty$ and $\mathbb{E}_{\mu}(Y)>-\infty$, then we have the following property

$$
\text { (sub-additivity) } \quad \mathbb{E}_{\mu}(X+Y) \leq \mathbb{E}_{\mu}(X)+\mathbb{E}_{\mu}(Y) .
$$

Remark 2.2 We refer the reader to Denneberg (1994) for a slightly weaker assumption than the one given in the previous proposition.

Remark 2.3 The reader should not be misled by the vocabulary used in the paper. We emphasize that when the capacity $\mu$ is concave in the sense of definition 2.5 , the functional $\mathbb{E}_{\mu}($.$) is a$ convex functional on $\chi$ (in the usual sense).

Other well-known results about Choquet integrals, quantile functions with respect to a capacity and comonotonic functions which will be used in the sequel can be found in the Appendix A.

We end this section by two examples of a capacity. The first example is well-known in the decision theory (think for instance of the rank-dependent expected utility theory - Quiggin 1982 or of the Yarii's distorted utility theory in Yaari 1997); the second is a slight generalization of the first.

1. Let $\mu$ be a probability measure on $(\Omega, \mathcal{F})$ and let $\psi:[0,1] \rightarrow[0,1]$ be a non-decreasing function on $[0,1]$ such that $\psi(0)=0$ and $\psi(1)=1$. Then the set function $\psi \circ \mu$ defined by $\psi \circ \mu(A):=\psi(\mu(A)), \forall A \in \mathcal{F}$ is a capacity in the sense of definition 2.1. The function $\psi$ is called a distortion function and the capacity $\psi \circ \mu$ is called a distorted probability. If the distortion function $\psi$ is concave, the capacity $\psi \circ \mu$ is a concave capacity in the sense of definition 2.5.

2. Let $\mu$ be a capacity on $(\Omega, \mathcal{F})$ and let $\psi$ be a distortion function. Then the set function $\psi \circ \mu$ is a capacity which, by analogy to the previous example, will be called a distorted capacity. Moreover, we have the following property : if $\mu$ is a concave capacity and $\psi$ is concave, then $\psi \circ \mu$ is concave. The proof uses the same arguments as the proof of proposition 4.69 in Föllmer and Schied (2004) and is left to the reader (see also exercice 2.10 in Denneberg 1994). 


\section{Stochastic orderings with respect to a capacity}

The concept of stochastic dominance is a well-known and useful concept in decision theory. It consists of introducing a partial order in the space of random variables on some probability space $(\Omega, \mathcal{F}, \mathbb{P})$ (more precisely, stochastic dominance relations are partial order relations on the set of the corresponding distribution functions). The aim of this section is to "extend" the concept of stochastic orderings to the case where the probability $\mathbb{P}$ is replaced by the more general notion of capacity; for the purposes of this article, the stress is placed on the generalizations of the results on the increasing convex and the increasing concave stochastic dominance relations to the case of a capacity. As usually done in the classical case, we emphasize the links between an economic approach to stochastic orderings based on numerical representations of the economic agents' preferences and a statistical approach based on a pointwise comparison of the distribution functions or of some other performance functions constructed from the distribution functions. Our definitions are analogous to the "classical" case of a probability measure.

\subsection{The increasing convex stochastic dominance with respect to a ca- pacity $\mu$}

Analogously to the "classical" definition of an increasing convex stochastic dominance (with respect to a probability measure), we define the notion of an increasing convex stochastic dominance relation (or equivalently an increasing convex ordering) with respect to a capacity $\mu$ as follows:

Definition 3.1 Let $X$ and $Y$ be two measurable functions on $(\Omega, \mathcal{F})$ and let $\mu$ be a capacity on $(\Omega, \mathcal{F})$. We say that $X$ is smaller than $Y$ in the increasing convex ordering (with respect to the capacity $\mu$ ) denoted by $X \leq_{i c x} Y$ if

$$
\mathbb{E}_{\mu}(u(X)) \leq \mathbb{E}_{\mu}(u(Y))
$$

for all functions $u: \mathbb{R} \rightarrow \mathbb{R}$ which are non-decreasing and convex, provided the Choquet integrals exist in $\mathbb{R}$.

This definition coincides with the usual definition of the increasing convex order when the capacity $\mu$ is a probability measure on $(\Omega, \mathcal{F})$. (cf. Shaked and Shanthikumar 2006 for details in the classical case) 
Remark 3.1 The economic interpretation of the $i c x$ ordering with respect to a capacity $\mu$ is the following: $X \leq_{i c x} Y$ if all the agents whose preferences are described by the (common) capacity $\mu$ and a non-decreasing convex utility function prefer the claim $Y$ to the claim $X$. As explained in Kaas et al. (2001), the "classical" stochastic orderings allow to compare risks (or financial positions) according to the expected utility (EU) paradigm. The stochastic orderings with respect to a capacity studied here allow to compare financial positions according to the Choquet expected utility (CEU) theory. The $\leq_{i c x, \mu}$ relation and the $\leq_{i c v, \mu}$ relation (defined below) derive from the CEU theory as the corresponding "classical" stochastic orderings derive from the EU model.

Let us mention that an economic setting where all the agents are CEU-maximizers characterized by a common capacity $\mu$ and a non-decreasing convex (resp. concave) utility function has already been considered in the literature in the study of Pareto-optima (cf. Chateauneuf et al. 2000).

For the sake of completeness, we define the notion of an increasing concave stochastic dominance (or equivalently an increasing concave ordering) with respect to a capacity $\mu$.

Definition 3.2 Let $X$ and $Y$ be two measurable functions on $(\Omega, \mathcal{F})$ and let $\mu$ be a capacity on $(\Omega, \mathcal{F})$. We say that $X$ is smaller than $Y$ in the increasing concave ordering (with respect to the capacity $\mu$ ) denoted by $X \leq_{i c v} Y$ if

$$
\mathbb{E}_{\mu}(u(X)) \leq \mathbb{E}_{\mu}(u(Y))
$$

for all functions $u: \mathbb{R} \rightarrow \mathbb{R}$ which are non-decreasing and concave, provided the Choquet integrals exist in $\mathbb{R}$.

Remark 3.2 As in the previous section, the dependence on the capacity $\mu$ in the notation for the stochastic dominance relations $\leq_{i c x}$ and $\leq_{i c v}$ is intentionally omitted. Nevertheless, we shall note $\leq_{i c x, \mu}$ and $\leq_{i c x, \mu}$ when an explicit mention of the capacity to which we refer is needed.

As in the classical case where the capacity $\mu$ is a probability measure, the ordering relations $\leq_{i c x}$ and $\leq_{i c v}$ are linked to each other in the following manner:

Proposition 3.1 Let $X$ and $Y$ be two measurable functions. Then

$$
X \leq_{i c x, \mu} Y \Leftrightarrow-Y \leq_{i c v, \bar{\mu}}-X
$$


where $\bar{\mu}$ denotes the dual capacity of the capacity $\mu$

(the dual capacity $\bar{\mu}$ being defined by $\left.\bar{\mu}(A)=1-\mu\left(A^{c}\right), \forall A \in \mathcal{F}\right)$.

Proof: The proof is based on the fact that a function $x \mapsto u(x)$ is non-decreasing and convex in $x$ if and only if the function $x \mapsto-u(-x)$ is non-decreasing and concave in $x$ and on the property of asymmetry of the Choquet integral; the details are straightforward.

Notice that in the classical case where the capacity $\mu$ is a probability measure, the dual capacity is the probability itself and so proposition 3.1 reduces to a well-known result from the stochastic order literature.

The aim of the following propositions is to obtain characterizations of the stochastic dominance relations $\leq_{i c x}$ and $\leq_{i c v}$. Due to proposition 3.1, we need to consider the case of $\leq_{i c x}$ only.

Proposition 3.2 Let $\mu$ be a capacity. Then we have the following statements:

(i) If $X \leq_{\mathrm{icx}, \mu} Y$, then $\mathbb{E}_{\mu}\left((X-b)^{+}\right) \leq \mathbb{E}_{\mu}\left((Y-b)^{+}\right), \forall b \in \mathbb{R}$, provided the Choquet integrals exist in $\mathbb{R}$.

(ii) If the capacity $\mu$ has the additional properties of continuity from below and continuity from above, then the converse implication holds true, namely, if $\mathbb{E}_{\mu}\left((X-b)^{+}\right) \leq \mathbb{E}_{\mu}\left((Y-b)^{+}\right), \forall b \in \mathbb{R}$, provided the Choquet integrals exist in $\mathbb{R}$, then $X \leq_{\mathrm{icx}, \mu} Y$.

Proof: The proof adapts the proof of theorem 1.5.7. in Müller and Stoyan (2002) to our case.

The proof of assertion (i) is trivial, the function $x \mapsto(x-b)^{+}$being non-decreasing and convex for all $b \in \mathbb{R}$.

Let us now prove the assertion (ii). Let $u$ be a non-decreasing and convex function (such that $\mathbb{E}_{\mu}(u(X))$ exists in $\mathbb{R}$ and $\mathbb{E}_{\mu}(u(Y))$ exists in $\left.\mathbb{R}\right)$. We consider three cases:

1. $\lim _{x \rightarrow-\infty} u(x)=0$. Then it is well-known that $u$ can be approximated from below by a sequence $\left(u_{n}\right)$ of functions of the following form (cf. Müller and Stoyan 2002):

$$
u_{n}(x)=\sum_{i=1}^{n} a_{i n}\left(x-b_{i n}\right)^{+}
$$


where $a_{i n} \geq 0$ and $b_{i n} \in \mathbb{R}$. Let us now remark that all the functions in the family $\left(a_{i n}\left(X-b_{i n}\right)^{+}\right)_{i \in\{1, \cdots, n\}}$ are pairwise comonotonic (thanks to proposition A.2) and so for all $i \in\{2, \cdots, n\}, a_{i n}\left(X-b_{i n}\right)^{+}$is comonotonic with $\sum_{j=1}^{i-1} a_{j n}\left(X-b_{j n}\right)^{+}$. Hence, using the additivity of the Choquet integral with respect to comonotonic functions and the positive homogeneity of the Choquet integral, we obtain

$$
\mathbb{E}_{\mu}\left(u_{n}(X)\right)=\sum_{i=1}^{n} a_{i n} \mathbb{E}_{\mu}\left[\left(X-b_{i n}\right)^{+}\right] \leq \sum_{i=1}^{n} a_{i n} \mathbb{E}_{\mu}\left[\left(Y-b_{i n}\right)^{+}\right]=\mathbb{E}_{\mu}\left(u_{n}(Y)\right) .
$$

The capacity $\mu$ being continuous from below, we apply the monotone convergence theorem as stated in theorem A.1 in order to pass to the limit in the previous inequality and to obtain

$$
\mathbb{E}_{\mu}(u(X)) \leq \mathbb{E}_{\mu}(u(Y))
$$

2. The case when $\lim _{x \rightarrow-\infty} u(x)=a \in \mathbb{R}$ can be reduced to the previous one by considering the function $x \mapsto u(x)-a$. Thus, we obtain that $\mathbb{E}_{\mu}(u(X)-a) \leq \mathbb{E}_{\mu}(u(Y)-a)$ and conclude thanks to the translation invariance of the Choquet integral.

3. If $\lim _{x \rightarrow-\infty} u(x)=-\infty$, then the function $u_{n}(x):=\max (u(x),-n)$ fulfils the conditions of the second case for any $n \in \mathbb{N}$ (indeed, $u_{n}$ is non-decreasing, convex and bounded from below), so

$$
\mathbb{E}_{\mu}\left(u_{n}(X)\right) \leq \mathbb{E}_{\mu}\left(u_{n}(Y)\right), \forall n \in \mathbb{N}
$$

Moreover, $u_{n}$ decreases to $u$, or equivalently, $-u_{n}$ increases to $-u$. A natural idea is then to apply the monotone convergence theorem in order to pass to the limit in the previous inequality by using the asymmetry of the Choquet integral and by observing that the continuity from above of $\mu$ is equivalent to the continuity from below of $\bar{\mu}$.

Note that the previous reasoning is rigourous in the case where we restrain ourselves to functions in $\chi$. Indeed, if $X$ is a function in $\chi$ (hence $X$ is bounded), then $\left(u_{n}(X)\right)$ is a bounded sequence (in fact, it is easily seen that $\max (u(\sup X), 0) \geq u_{n}(X) \geq u(\inf X)$ for all $n$ where $\inf X$ and $\sup X$ denote the lower and upper bound of $X$ respectively). Therefore, the monotone convergence theorem as stated in theorem A.1 combined with the translation invariance of the Choquet integral allows us to conclude.

In the general case, the sequence of functions $\left(-u_{n}(X)\right)$ is not necessarily bounded from below and we cannot apply the monotone convergence theorem (theorem A.1). Nevertheless, 
by the same arguments as in the proof of theorem 8.1 in Denneberg (1994) (see also the proof of lemma C.1 in the appendix), we see that if a sequence of real-valued measurable functions $\left(Z_{n}\right)$ converges from below to a real-valued function $Z$ (denoted by $Z_{n} \uparrow Z$ ) and if a capacity $\nu$ is continuous from below, then the sequence of distribution functions $\left(G_{Z_{n}, \nu}\right)$ (with respect to $\nu$ ) converges monotonely to the distribution function $G_{Z, \nu}$ of $Z$. So, the corresponding sequence of quantile functions $\left(r_{Z_{n}, \nu}\right)$ converges from below to (the) quantile function $r_{Z, \nu}$ of $Z$ almost everywhere (see the proof of lemma C.2 in the appendix for the same argument). Therefore, we can use proposition A.1 and the dominated convergence theorem for Lebesgue integrals (with respect to the Lebesgue measure on $[0,1]$ ) in order to conclude. Indeed, it suffices to set $Z_{n}:=-u_{n}(X), Z:=-u(X)$ and $\nu:=\bar{\mu}$ and to observe that $r_{Z_{0}, \nu} \leq r_{Z_{n}, \nu} \leq r_{Z, \nu}$ almost everywhere and that the functions $r_{Z, \nu}$ and $r_{Z_{0}, \nu}$ are integrable with respect to the Lebesgue measure by assumption.

We give some details concerning the integrability of $r_{Z, \nu}$ and $r_{Z_{0}, \nu}$ for reader's convenience. Being a non-decreasing function on $(0,1)$, the function $r_{Z, \nu}$ is integrable in the Lebesgue sense if and only if its generalized Riemann integral exists and is finite. Thus, the integrability of $r_{Z, \nu}$ is due to the equation

$$
\int_{0}^{1} r_{Z, \nu} d t=\int_{0}^{1} r_{Z, \bar{\mu}} d t=\mathbb{E}_{\bar{\mu}}(Z)=\mathbb{E}_{\bar{\mu}}(-u(X))=-\mathbb{E}_{\mu}(u(X)),
$$

where the term $\mathbb{E}_{\mu}(u(X))$ belongs to $\mathbb{R}$ by assumption. The integrability of $r_{Z_{0}, \nu}$ is a consequence of that of $r_{Z, \nu}$ and can be proved by means of similar calculations.

Observe that in the classical case where $\mu$ is a probability measure the previous proposition reduces to a well-known characterization of the increasing convex order; it allows to link the increasing convex order to the notion of a stop-loss premium in reinsurance. Accordingly, in the classical case, the increasing convex order is sometimes called stop-loss order.

Let us now establish a link between the increasing convex stochastic dominance with respect to a capacity $\mu$ and the distribution function with respect to the capacity $\mu$.

Proposition 3.3 Let $\mu$ be a capacity and let $X$ and $Y$ be two measurable functions. The following two statements are equivalent: 
(i) $\mathbb{E}_{\mu}\left((X-b)^{+}\right) \leq \mathbb{E}_{\mu}\left((Y-b)^{+}\right), \forall b \in \mathbb{R}$, provided the Choquet integrals exist in $\mathbb{R}$.

(ii) $\int_{x}^{+\infty} \mu(X>u) d u \leq \int_{x}^{+\infty} \mu(Y>u) d u, \forall x \in \mathbb{R}$, provided the integrals exist in $\mathbb{R}$.

Proof: Using the definition of the Choquet integral and a change of variables, we have for all $b \in \mathbb{R}$,

$$
\mathbb{E}_{\mu}\left((X-b)^{+}\right)=\int_{0}^{+\infty} \mu\left((X-b)^{+}>u\right) d u=\int_{0}^{+\infty} \mu(X>u+b) d u=\int_{b}^{+\infty} \mu(X>u) d u
$$

which proves the desired result.

Now, we are ready to relate the previous results to the notion of a quantile function with respect to $\mu$. We refer the reader to Shaked and Shanthikumar (2006) for a proof of the following result in the classical case of a probability measure and to Ogryczak and Ruszczynski (2001) for a different proof of the same result based on convex duality; see also lemma A.22 in Föllmer and Schied (2004). Our proof is inspired by the last two references.

Proposition 3.4 Let $\mu$ be a capacity and let $X$ and $Y$ be two real-valued measurable functions such that $\int_{0}^{1}\left|r_{X}(t)\right| d t<+\infty$ and $\int_{0}^{1}\left|r_{Y}(t)\right| d t<+\infty$ where $r_{X}$ and $r_{Y}$ denote (the) quantile functions of $X$ and $Y$ with respect to $\mu$. The following two statements are equivalent:

(i) $G_{X}^{(2)}(x):=\int_{x}^{+\infty} \mu(X>u) d u \leq \int_{x}^{+\infty} \mu(Y>u) d u=: G_{Y}^{(2)}(x), \forall x \in \mathbb{R}$.

(ii) $\int_{y}^{1} r_{X}(t) d t \leq \int_{y}^{1} r_{Y}(t) d t, \forall y \in[0,1]$.

In order to prove the proposition we need the following lemma which corresponds to lemma A.22 in Föllmer and Schied (2004) in the classical case.

Lemma 3.1 Let $\mu$ be a capacity on $(\Omega, \mathcal{F})$ and let $X$ be a measurable function on $(\Omega, \mathcal{F})$ such that the quantile function $r_{X}$ of $X$ with respect to $\mu$ is integrable (with respect to the Lebesgue measure on $[0,1])$. Define the function $G_{X}^{(2)}$ by

$$
G_{X}^{(2)}(x):=\int_{x}^{+\infty} \mu(X>u) d u=\int_{x}^{+\infty}\left(1-G_{X}(u)\right) d u, x \in \mathbb{R} .
$$


Then the conjugate function of $G_{X}^{(2)}$ is given by

$$
r_{X}^{(2)}(y):=\sup _{x \in \mathbb{R}}\left(x y-G_{X}^{(2)}(x)\right)=\left\{\begin{array}{l}
-\int_{y+1}^{1} r_{X}(t) d t, \text { if } y \in[-1,0] \\
+\infty, \text { otherwise. }
\end{array}\right.
$$

Proof of the lemma: The arguments of the proof being almost the same as those of Föllmer and Schied (2004), the proof is placed in the Appendix B.

We are ready to prove proposition 3.4.

\section{Proof of proposition 3.4:}

The proof is based on lemma 3.1 .

Suppose that (i) holds true i.e. $G_{X}^{(2)}(x) \leq G_{Y}^{(2)}(x), \forall x \in \mathbb{R}$. Then for all $y \in \mathbb{R}$,

$$
r_{X}^{(2)}(y):=\sup _{x \in \mathbb{R}}\left(x y-G_{X}^{(2)}(x)\right) \geq \sup _{x \in \mathbb{R}}\left(x y-G_{Y}^{(2)}(x)\right)=: r_{Y}^{(2)}(y)
$$

which implies, in particular, that $-\int_{y+1}^{1} r_{X}(t) d t \geq-\int_{y+1}^{1} r_{Y}(t) d t$, for all $y \in[-1,0]$, or equivalently,

$$
\int_{y}^{1} r_{X}(t) d t \leq \int_{y}^{1} r_{Y}(t) d t, \text { for all } y \in[0,1]
$$

The converse implication can be obtained by means of a similar argument after observing that the function $G_{X}^{(2)}$ is the conjugate function of $r_{X}^{(2)}$. Indeed, this follows from the fact that the function $G_{X}^{(2)}$ is convex, proper and lower-semicontinuous (cf. theorem 24.2 in Rockafellar 1972) and from the biduality theorem (cf. theorem 12.2 in Rockafellar 1972).

We conclude this section by establishing another useful characterization of the relation $\leq_{i c x}$ which will be needed in the sequel. Its analogue in the classical case of a probability measure is due to Dana (2005) (see also thm. 5.2.1 in Dhaene et al. 2006 for a related result). Our proof follows the proof of the former.

Proposition 3.5 Let $X \in \chi$ and $Y \in \chi$ be given. Then the following statements are equivalent:

(i) $\int_{y}^{1} r_{X}(t) d t \geq \int_{y}^{1} r_{Y}(t) d t, \forall y \in[0,1]$

(ii) $\int_{0}^{1} g(t) r_{X}(t) d t \geq \int_{0}^{1} g(t) r_{Y}(t) d t, \forall g:[0,1] \rightarrow \mathbb{R}_{+}$, integrable, non-decreasing.

Proof: Being quite similar to the proof of Dana (2005), the proof is given in the Appendix B. 
Remark 3.3 An economic interpretation of the $\leq_{i c x, \mu}$-relation in terms of "uniform" preferences is given in remark 3.1 ; the interpretation is based on the initial definition of the $\leq_{i c x, \mu^{-}}$ relation (definition 3.1).

An interpretation of the $\leq_{i c x, \mu}$-relation in terms of ambiguity is suggested by the equivalence established in proposition 3.3. Indeed, let us first consider the inequality $\mu(X>t) \geq \mu(Y>t)$ where $t \in \mathbb{R}$ is fixed. Bearing in mind that the capacity $\mu$ models the agent's perception of "uncertain" (or "ambiguous") events, the reader may interpret the previous inequality as having the following meaning: the event $\{X>t\}$ is perceived by the agent as being less uncertain than or equally uncertain to the event $\{Y>t\}$. Then, part (ii) in proposition 3.3 may be loosely read as follows: the agent "feels less or equally uncertain about the financial position $X$ 's taking great values on average than the financial position $Y$ 's".

\section{A useful tool: the generalized Hardy-Littlewood's inequal- ities}

In this section we state a useful result which can be seen as a "generalization" of the well-known Hardy-Littlewood's inequalities to the present setting.

For the statement and the proof of this result in the classical case of a probability measure we refer to theorem A. 24 in Föllmer and Schied (2004); some applications of the "classical" HardyLittlewood's inequalities to finance can be found in the same reference. Other applications of the "classical" version to economics and finance can be found in Carlier and Dana (2006); see also Carlier and Dana (2005) (and references therein) where a supermodular extension of the "classical" inequalities is used in insurance.

The generalization that we state in this section will be needed while dealing with the optimization problem of the following section. This generalized version proves to be useful in our ongoing work concerning some static optimization problems related to the CEU theory (cf. Grigorova 2010).

Proposition 4.1 (Hardy-Littlewood's inequalities) Let $\mu$ be a capacity on $(\Omega, \mathcal{F})$. Let $X$ and $Y$ be two non-negative measurable functions with quantile functions (with respect to the capacity $\mu$ ) denoted by $r_{X}$ and $r_{Y}$. 
1. If $\mu$ is concave and continuous from below, then

$$
\mathbb{E}_{\mu}(X Y) \leq \int_{0}^{1} r_{X}(t) r_{Y}(t) d t
$$

2. If $\mu$ is convex and continuous from below, then

$$
\mathbb{E}_{\mu}(X Y) \geq \int_{0}^{1} r_{X}(1-t) r_{Y}(t) d t
$$

Proof: As the proof of this result is relatively long, it is placed in the Appendix C.

\section{Application to a financial optimization problem}

This section is devoted to the following optimization problem:

$$
\begin{gathered}
\text { Maximize } \mathbb{E}_{\mu}(Z C) \\
\text { under the constraints } C \in \chi_{+} \text {s.t. } C \leq_{i c x, \mu} X
\end{gathered}
$$

where $\chi_{+}$denotes the set of non-negative bounded measurable functions, $\mu$ is a given capacity, $Z$ is a given function in $\chi_{+}$and $X$ is a given function in $\chi_{+}$.

The study of this problem has been inspired by the work of Dana (2005) in the classical case of a probability measure (see Dana 2005 and references therein; see also Dana and Meilijson 2003, Jouini and Kallal 2001 and Dybvig 1987). Dana (2005) considers a similar problem to the stated above, namely,

$$
\text { Minimize } \mathbb{E}(Z C)
$$

$$
\text { under the constraints } C \in L^{\infty}(\mathbb{P}) \text { s.t. } X \leq_{i c v} C
$$

where $\mathbb{E}$ denotes expectation with respect to $\mathbb{P}$ and $\leq_{i c v}$ denotes the increasing concave order relation in the classical sense. The problem $(\tilde{D})$ has the following economic interpretation: the measurable function $Z$ being interpreted as a pricing kernel (in the case where $\mathbb{E}(Z)=1$ ), the problem is to find the contingent claim $C$ with minimal price among all contingent claims which dominate the claim $X$ in the increasing concave order, or equivalently, among all the claims $C$ which are preferred to $X$ by all the investors whose preferences are described by a non-decreasing and concave utility function.

An analogous interpretation may be given in the setting of problem (D). Indeed, let us place ourselves in a world where the agents are facing "ambiguous events" and let us assume that all 
the agents perceive ambiguity in the same manner i.e. through the same capacity $\mu$. In the case where the capacity $\mu$ is concave (which will be the case later on), the objective functional $C \mapsto \mathbb{E}_{\mu}(Z C)$ can be interpreted as a non-additive pricing functional which has the properties of monotonicity and convexity and the non-negative measurable function $Z$ can be seen as a discount factor or, more generally, a "change of numéraire". A pricing rule of this form (in the case $Z \equiv 1$ ) is used in Chateauneuf et al. (2000) in order to model the selling price of a claim (its buying price being modelled by $\left.E_{\bar{\mu}}().\right)$. Thus, the problem (D) consists in finding a contingent claim $C$ having the maximal price among all the non-negative contingent claims which are dominated by $X$ in the increasing convex stochastic dominance (with respect to the capacity $\mu$ ).

Adopting the terminology introduced by Jouini and Kallal (2001), we may call the value function $e(X, Z)$ of problem (D) (when $Z$ is fixed) the "utility price" of $X$ in the context of ambiguity. It will be shown in subsection 5.2 that, for a fixed $Z$, the utility price in the context of ambiguity $e(., Z)$ is the smallest functional on $\chi_{+}$among those which are consistent with respect to the $\leq_{i c x, \mu}$-relation and which are greater than or equal to the "market price" $\mathbb{E}_{\mu}(Z$.).

We have the following theorem which is the analogue of theorem 2.1 in Dana (2005).

Theorem 5.1 Let $\mu$ be a concave and continuous from below capacity. For every function $X \in$ $\chi_{+}$and for every function $Z \in \chi_{+}$such that the distribution function $G_{Z}$ of $Z$ with respect to $\mu$ is continuous, the problem

$$
\text { Maximize } \mathbb{E}_{\mu}(Z C)
$$

$$
\text { under the constraints } C \in \chi_{+} \text {s.t. } C \leq_{\mathrm{icx}, \mu} X
$$

has a solution and its value function $e(X, Z)$ is given by:

$$
e(X, Z)=\int_{0}^{1} r_{Z}(t) r_{X}(t) d t .
$$

Proof: We have

$$
\begin{aligned}
e(X, Z) & =\sup _{0 \leq C, C \leq i c x, \mu} \mathbb{E}_{\mu}(Z C) \leq \sup _{0 \leq C, C \leq i c x, \mu} \int_{0}^{1} r_{Z}(t) r_{C}(t) d t \\
& \leq \int_{0}^{1} r_{Z}(t) r_{X}(t) d t
\end{aligned}
$$

where the first inequality is due to the upper bound in Hardy-Littlewood's inequalities (proposition 4.1), the second inequality is a consequence of proposition 3.5 (applied with $g=r_{Z}$ ). 
Thus we obtain that $e(X, Z) \leq \int_{0}^{1} r_{Z}(t) r_{X}(t) d t$. To conclude we need to find $C \in \chi_{+}$such that $C \leq_{i c x, \mu} X$ and such that $\mathbb{E}_{\mu}(Z C)=\int_{0}^{1} r_{Z}(t) r_{X}(t) d t$.

Set $f(x):=r_{X}\left(G_{Z}(x)\right)$, then $C:=f(Z)$ is as wanted. Indeed, $C \geq 0$. Moreover,

$$
\mathbb{E}_{\mu}(Z C)=\mathbb{E}_{\mu}(Z f(Z))=\mathbb{E}_{\mu}(h(Z))=\int_{0}^{1} r_{h(Z)}(t) d t
$$

where we have used proposition A.1 in the last equality and where $h: \mathbb{R}_{+} \rightarrow \mathbb{R}_{+}$is defined by $h(z):=z f(z), \forall z \geq 0$. The function $h$ being non-decreasing and the function $G_{Z}$ being continuous by assumption, we can apply lemma A.1 to obtain

$$
\begin{aligned}
\mathbb{E}_{\mu}(Z C) & =\int_{0}^{1} h\left(r_{Z}(t)\right) d t=\int_{0}^{1} r_{Z}(t) f\left(r_{Z}(t)\right) d t \\
& =\int_{0}^{1} r_{Z}(t) r_{X}\left(G_{Z}\left(r_{Z}(t)\right)\right) d t=\int_{0}^{1} r_{Z}(t) r_{X}(t) d t .
\end{aligned}
$$

where we have used the continuity of $G_{Z}$ in the last step.

We are left with establishing that $f(Z) \leq_{i c x, \mu} X$. We will check this property using the definition of $\leq_{i c x, \mu}$. Let $u$ be a non-decreasing, convex function. We have

$$
\mathbb{E}_{\mu}(u(f(Z)))=\int_{0}^{1} r_{u(f(Z))}(t) d t=\int_{0}^{1} u\left(f\left(r_{Z}(t)\right)\right) d t
$$

where the second equality follows from lemma A.1 (the function $u \circ f$ being non-decreasing and the function $G_{Z}$ being continuous by assumption). This gives

$$
\begin{aligned}
\mathbb{E}_{\mu}(u(f(Z))) & =\int_{0}^{1} u\left(r_{X}\left(G_{Z}\left(r_{Z}(t)\right)\right)\right) d t=\int_{0}^{1} u\left(r_{X}(t)\right) d t \\
& =\int_{0}^{1} r_{u(X)}(t) d t=\mathbb{E}_{\mu}(u(X))
\end{aligned}
$$

where the last but one equality is obtained thanks to lemma A.1 after observing that $u$ is a continuous function as a real-valued convex function on $\mathbb{R}$.

This concludes the proof.

Remark 5.1 The previous proof can be extended to the case where the assumption of the boundedness from above of $Z$ is replaced by the weaker assumption that $\int_{0}^{1}\left|r_{Z}(t)\right| d t<+\infty$. This is due mainly to proposition 3.5 where only the non-negativity and the integrability of $r_{Z}$ are required. We have nevertheless chosen to present the previous result in the case where all the functions are in $\chi$. 
In the classical case where $\mu$ is a probability measure the result of theorem 5.1 still holds even when the continuity assumption on the distribution function $G_{Z}$ of $Z$ is relaxed. More precisely, we have the following result:

Proposition 5.1 Let $\mu$ be a probability measure on $(\Omega, \mathcal{F})$. For every function $X \in \chi_{+}$and for every function $Z \in \chi_{+}$, the problem

\section{Maximize $\mathbb{E}(Z C)$}

under the constraints $C \in \chi_{+}$s.t. $C \leq_{\mathrm{icx}} X$

has a solution and its value function is given by $\int_{0}^{1} r_{Z}(t) r_{X}(t) d t$.

The symbol $\mathbb{E}$ denotes the (classical) expectation with respect to $\mu$ and $\leq_{\mathrm{icx}}$ denotes the (classical) increasing convex stochastic dominance relation with respect to $\mu$.

Proof: We sketch the proof following the proof of theorem 5.1 and stressing only on the changes to be made in the proof of theorem 5.1. Note that applying lemma A.1 is still possible whenever needed in this case (even without the continuity assumption on $G_{Z}$ ) thanks to remark A.1. Nevertheless, the continuity of $G_{Z}$ being used to obtain the last equality in equation (5.1), the function $f$ in the proof of theorem 5.1 is now replaced by the function $\tilde{f}$ defined by $\tilde{f}(x):=$ $r_{X}\left(G_{Z}(x)\right)$ if $x$ is a continuity point of $G_{Z}$ and by $\tilde{f}(x):=\frac{1}{G_{Z}(x)-G_{Z}(x-)} \int_{G_{Z}(x-)}^{G_{Z}(x)} r_{X}(t) d t$ if $x$ is not a continuity point of $G_{Z}$. The function $\tilde{f}$ is non-decreasing and satisfies the property $\tilde{f}\left(r_{Z}\right)=\mathbb{E}_{\lambda}\left(r_{X} \mid r_{Z}\right)$ where the symbol $\mathbb{E}_{\lambda}(. \mid$.) denotes the conditional expectation with respect to the Lebesgue measure $\lambda$.

We set $\tilde{h}(x):=x \tilde{f}(x)$ and we replace equation (5.1) by the following

$$
\begin{aligned}
\mathbb{E}(Z C) & =\int_{0}^{1} \tilde{h}\left(r_{Z}(t)\right) d t=\int_{0}^{1} r_{Z}(t) \tilde{f}\left(r_{Z}(t)\right) d t \\
& =\int_{0}^{1} r_{Z}(t) r_{X}(t) d t .
\end{aligned}
$$

where lemma A.1 and remark A.1 are used to obtain the first equality and the characterization of the conditional expectation is used to obtain the last.

Equation (5.2) remains unchanged, the function $f$ being replaced by the function $\tilde{f}$; we have again applied lemma A.1 and remark A.1 to obtain it. 
Equation (5.3) has to be replaced by

$$
\begin{aligned}
\mathbb{E}(u(f(Z))) & =\int_{0}^{1} u\left(\tilde{f}\left(r_{Z}(t)\right)\right) d t \leq \int_{0}^{1} u\left(r_{X}(t)\right) d t \\
& =\int_{0}^{1} r_{u(X)}(t) d t=\mathbb{E}(u(X))
\end{aligned}
$$

where we have applied Jensen's inequality.

Remark 5.2 Note that in the case where the underlying probability space $(\Omega, \mathcal{F}, \mu)$ is atomless the use of lemma A.1 (and remark A.1) in the previous proof can be replaced by the use of the following two usual arguments: the law invariance of the functional $\mathbb{E}(l()):. \chi_{+} \rightarrow \mathbb{R}_{+}$where $l: \mathbb{R}_{+} \rightarrow \mathbb{R}_{+}$is a measurable function and the fact that the law of $Z$ is the same as the law of $r_{Z}(U)$ where $U$ denotes a uniform random variable on $(0,1)$. Then, the above proof is almost the same as the proof of theorem 2.1 in Dana (2005) (see also Dana and Meilijson 2003 and Föllmer and Schied 2004). We note that the use of lemma A.1 in the proof of proposition 5.1 provides an alternative argument to the "law-invariance argument" even beyond the nonatomic case.

Remark 5.3 Let us mention that, thanks to remark A.1, the continuity assumption on $G_{Z}$ in theorem 5.1 may be relaxed in the case of a capacity $\mu$ which, apart from the properties required in theorem 5.1, has the additional property of continuity from above.

Let us further note that for a concave capacity $\mu$ (which is the case in theorem 5.1) the property of continuity from above of $\mu$ implies the property of continuity from below.

\subsection{The value function of problem (D) as a risk measure}

While studying the problem $(\tilde{D})$ in the classical setting, Dana (2004) gives an interpretation of its value function in terms of risk measures. An analogous commentary can be made in the present setting.

Consider the value function $e(., Z)$ of problem (D) for a fixed $Z$ as a functional of the first argument and extend it to the whole set $\chi$. More precisely, let us consider the functional $\rho: \chi \rightarrow$ $\mathbb{R}$ defined by $\rho(X):=e(X, Z):=\int_{0}^{1} r_{Z}(t) r_{X}(t) d t$ where $Z$ is a fixed non-negative measurable function in $\chi$. For the easing of the presentation, we will assume in the rest of this section that $Z$ is such that $\int_{0}^{1} r_{Z, \mu}(t) d t=1$. This assumption is not a serious restriction because, due to the 
positive homogeneity of the objective functional of problem (D), we may as well replace $Z$ by $\frac{Z}{\int_{0}^{1} r_{Z, \mu}(t) d t}$ (in the case where $\int_{0}^{1} r_{Z, \mu}(t) d t \neq 0$ ) in the formulation of problem (D). The functional $\rho$ is monotone $(X \leq Y$ implies $\rho(X) \leq \rho(Y))$ and translation invariant $(\rho(X+b)=$ $\rho(X)+b, \forall b \in \mathbb{R})$. Therefore, according to the definition given in Artzner et al. (1999), up to a minus sign $\rho$ is a monetary measure of risk on $\chi$ (see also Wang and Yan (2007) or Ekeland et al. (2009) for the same "sign convention" as the one used in the present paper). Moreover, $\rho$ is additive with respect to comonotonic elements of $\chi$; this property is due to the comonotonic additivity of the quantile function with respect to a capacity. Monetary risk measures having the property of comonotonic additivity have already been studied in the literature (cf. Föllmer and Schied 2004), the idea being that when $X$ and $Y$ are comonotonic, $X$ cannot act as a hedge against $Y$. The risk measure $\rho$ has the additional property of being consistent with the increasing convex ordering relation $\leq_{i c x, \mu}$ which means that if $X \leq_{i c x, \mu} Y$ then $\rho(X) \leq \rho(Y)$. This consistency property is easily obtained thanks to proposition 3.5 when observing that the function $r_{Z}$ which stands in the place of the function $g$ of proposition 3.5 is non-negative and integrable.

Furthermore, the risk measure $\rho$ can be represented as a Choquet integral with respect to a certain capacity. Indeed, according to a well-known representation result for monotone and comonotonicly additive functionals on $\chi$ (cf. thm. 4.82. in Föllmer and Schied 2004 or Denneberg $1994)$ we know that there exists a capacity $\nu$ on $(\Omega, \mathcal{F})$ such that

$$
\rho(X)=\mathbb{E}_{\nu}(X) \text {, for all } X \in \chi \text {. }
$$

The capacity $\nu$ is related to the initial capacity $\mu$ in the following manner

$$
\nu(A)=\rho\left(\mathbb{I}_{A}\right)=e\left(\mathbb{I}_{A}, Z\right)=\int_{0}^{1} r_{Z, \mu}(t) r_{\mathbb{I}_{A}, \mu}(t) d t=\int_{1-\mu(A)}^{1} r_{Z, \mu}(t) d t, \forall A \in \mathcal{F} .
$$

Therefore, the capacity $\nu$ is of the form: $\nu(A)=\psi(\mu(A)), \forall A \in \mathcal{F}$ where $\psi(x):=\int_{1-x}^{1} r_{Z, \mu}(t) d t, \forall x \in$ $[0,1]$. We verify that the function $\psi$ is a distortion function in the sense of the definition given in section 2; hence, the capacity $\nu=\psi \circ \mu$ is a distorted capacity. Moreover, the distortion function $\psi$ being concave, $\nu$ is a concave capacity. Thus, the functional $\rho$ can be represented as a Choquet integral with respect to the concave distorted capacity $\psi \circ \mu$; hence, $\rho$ is a positively homogeneous, convex monetary measure of risk (or equivalently, a coherent monetary measure of risk in the terminology of Artzner et al. 1999). 
In fact, risk measures of the form $\mathbb{E}_{\psi \circ \mu}($.$) where \mu$ is a probability measure and $\psi$ is a (concave) distortion function have been studied by Wang et al. (1997) and Denneberg (1990) and are now known under the name of distortion risk measures or distortion premium principles (see, for instance, Dhaene et al. 2006 for a survey and examples). At the end of his article, Denneberg (1990) suggests possible generalizations to the case where the probability measure is replaced by a more general set function - the functional $\rho$ that we obtain could be seen as such a generalization. Adopting this point of view, we could call $\rho$ a "generalized" distortion risk measure.

Let us finally remark that the value function of problem (D) can be seen also as an analogue in the setting of ambiguity of the notion of maximal correlation risk measure (cf. Ekeland et al. 2009 and the references therein).

\subsection{The value function of problem (D) as a premium principle}

We give another interpretation of the value function of our problem (D) in terms of premium principles in insurance.

Consider an insurance company which uses a given premium principle as a reference but which is now willing to take into account other criteria of "riskiness" modelled through the stochastic dominance relation $\leq_{i c x, \mu}$. In insurance, elements of $\chi_{+}$are usually considered as payments which the company has to make (or losses it has to face) and premium principles are functionals on $\chi_{+}$ taking values in $\mathbb{R}$; these functionals are usually non-decreasing. In this framework, the objective functional of problem (D), namely the functional $\rho_{0}: \chi_{+} \mapsto \mathbb{R}_{+}$defined by $\rho_{0}(X):=\mathbb{E}_{\mu}(Z X)$, can be seen as the reference premium principle used by the company. We remark that the premium principle $\rho_{0}$ may be perceived as a kind of a generalization of the Esscher premium principle which is well-known in insurance (see for instance Young 2004 for a definition and other examples). In this context, the value function $e(., Z)$ of problem (D) is interpreted as a new premium principle which has (among other "desirable" properties) the property of consistency with respect to the relation $\leq_{i c x, \mu}$ and which is greater than or equal to the reference premium principle $\rho_{0}$ (i.e. $\left.e(X, Z) \geq \mathbb{E}_{\mu}(Z X), \forall X \in \chi_{+}\right)$. The latter property is due to the fact that $e(., Z)$ is the value function of problem (D) and to the reflexivity of the relation $\leq_{i c x, \mu}$.

Moreover, we have the following property:

Proposition 5.2 The value function e(., Z) of problem (D) is the smallest functional on $\chi_{+}$ 
which satisfies the property of consistency with respect to the relation $\leq_{\mathrm{icx}, \mu}$ and which is greater than or equal to $\rho_{0}$ where $\rho_{0}$ is given by $\rho_{0}(X):=\mathbb{E}_{\mu}(Z X), \forall X \in \chi_{+}$.

Proof: Let $F: \chi_{+} \mapsto \mathbb{R}$ be a functional which is consistent with $\leq_{i c x, \mu}$ and which is greater than or equal to $\rho_{0}$. For all $X \in \chi_{+}$and for all $C \in \chi_{+}$such that $C \leq_{i c x, \mu} X$, the property of consistency with respect to the relation $\leq_{i c x, \mu}$ implies that $F(X) \geq F(C)$. Moreover, $F(C) \geq$ $\mathbb{E}_{\mu}(Z C)$. So, by taking the supremum over the set $\left\{C \in \chi_{+}\right.$s.t. $\left.C \leq_{i c x, \mu} X\right\}$, we have $F(X) \geq$ $e(X, Z)$.

We conclude that the value function $e(., Z)$ of problem (D) is the smallest premium principle among those which are consistent with respect to the increasing convex dominance relation $\leq_{i c x, \mu}$ and which are greater than or equal to the initial premium principle $\rho_{0}$.

Thanks to the above considerations the insurance company may use problem (D) as a way of defining a new premium principle $e(., Z)$ on $\chi_{+}$(which premium principle induces a total preorder on $\chi_{+}$unlike the stochastic dominance relation $\leq_{i c x, \mu}$ which is only a partial pre-order). Loosely speaking, the newly obtained premium principle is "richer" than the initial premium principle $\rho_{0}$ because other criteria of "riskiness" and the "change of numéraire" $Z$ have been taken into account through problem (D).

\section{$6 \quad$ Future perspectives}

As seen in the previous section, a closely related question to the concepts studied in this article is the problem of risk measures respecting stochastic dominance relations. We are studying, in particular, the question of quantile-based risk measures with respect to a given capacity i.e. risk measures based on the quantile function $r_{X, \mu}$ where $\mu$ is a given capacity and where the measurable function $X$ models a financial position, and we are exploring their consistency with respect to the stochastic dominance relations defined above (cf. Grigorova 2010). 


\section{A Appendix: Some basic results about capacities and Cho- quet integrals}

The results of this appendix A can be found in the book by Föllmer and Schied (2004) (cf. section 4.7 of this reference) and/or in the one by Denneberg (1994) and are recalled here for reader's convenience.

\section{A.1 Choquet integrals and quantile functions}

We have the following well-known result where we make the convention that the assertion is valid provided the expressions make sense. The result can be found in Föllmer and Schied (2004) for the bounded case or deduced from Denneberg (1994) (cf. pages 61-62 in chapter 5 of the latter reference).

Proposition A.1 Let $X$ be a real-valued measurable function and let $r_{X}$ be a quantile function of $X$ with respect to a capacity $\mu$, then

$$
\mathbb{E}_{\mu}(X)=\int_{0}^{1} r_{X}(t) d t
$$

The following lemma is the analogue of lemma A.23. in Föllmer and Schied (2004) and can be found in Denneberg (1994).

Lemma A.1 Let $X=f(Y)$ where $f$ is a non-decreasing function and let $r_{Y}$ be a quantile function of $Y$ with respect to a capacity $\mu$. Suppose that $f$ and $G_{Y}$ have no common discontinuities, then $f \circ r_{Y}$ is a quantile function of $X$ with respect to $\mu$. In particular,

$$
r_{X}(t)=r_{f(Y)}(t)=f\left(r_{Y}(t)\right) \text { for almost every } t \in(0,1)
$$

where $r_{X}$ denotes a quantile function of $X$ with respect to $\mu$.

Remark A.1 If the capacity $\mu$ satisfies the additional properties of continuity from below and from above, the assumption of no common discontinuities of the functions $f$ and $G_{Y}$ can be dropped in the previous lemma. The proof is then analogous to the proof in the classical case of a probability measure (cf. lemma A.23. in Föllmer and Schied 2004 for a proof in the classical case) and is left to the reader. 


\section{A.2 A monotone convergence theorem for Choquet integrals}

We recall a monotone convergence theorem for Choquet integrals with respect to a capacity which is continuous from below; we refer the reader to Denneberg (1994) for a proof of this result.

Theorem A.1 (monotone convergence) Let $\mu$ be a capacity on $(\Omega, \mathcal{F})$ which is continuous from below. For a non-decreasing sequence $\left(X_{n}\right)$ of non-negative measurable functions, the limit function $X:=\lim _{n \rightarrow \infty} X_{n}$ is measurable and $\lim _{n \rightarrow \infty} \mathbb{E}_{\mu}\left(X_{n}\right)=\mathbb{E}_{\mu}(X)$.

\section{A.3 Comonotonic functions}

We have the following characterization of comonotonic functions which corresponds to proposition 4.5 in Denneberg (1994) (see also Föllmer and Schied 2004)

Proposition A.2 For two real-valued measurable functions $X, Y$ on $(\Omega, \mathcal{F})$ the following conditions are equivalent:

(i) $X$ and $Y$ are comonotonic.

(ii) There exists a measurable function $Z$ on $(\Omega, \mathcal{F})$ and two non-decreasing functions $f$ and $g$ on $\mathbb{R}$ such that $X=f(Z)$ and $Y=g(Z)$.

The notion of comonotonic functions proves to be very useful while dealing with Choquet integrals thanks to the following result (cf. lemma 4.84 in Föllmer and Schied 2004, as well as corollary 4.6 in Denneberg 1994).

Lemma A.2 If $X, Y: \Omega \rightarrow \mathbb{R}$ is a pair of comonotonic functions and if $r_{X}, r_{Y}, r_{X+Y}$ are quantile functions (with respect to a capacity $\mu$ ) of $X, Y, X+Y$ respectively, then

$$
r_{X+Y}=r_{X}+r_{Y}, \text { for almost every } t
$$

\section{B Appendix: The proofs of Lemma 3.1 and Proposition 3.5}

\section{Proof of lemma 3.1:}

Throughout this proof we set $\phi(x):=G_{X}^{(2)}(x)$ to alleviate the notations. Correspondingly, we 
denote by $\phi^{*}$ the conjugate function of $\phi$ i.e. $\phi^{*}(y):=\sup _{x \in \mathbb{R}}(x y-\phi(x))$.

Let us first remark that

$$
\phi(x)=\int_{x}^{+\infty} \mu(X>u) d u=\mathbb{E}_{\mu}\left((X-x)^{+}\right)=\int_{0}^{1}\left(r_{X}(t)-x\right)^{+} d t,
$$

the second equality is the straightforward transformation used in the proof of proposition 3.3 and the third is due to proposition A.1 and to lemma A.1.

Therefore, for $y=0$, we have

$$
\phi^{*}(0)=-\inf _{x \in \mathbb{R}} \int_{0}^{1}\left(r_{X}(t)-x\right)^{+} d t=-\lim _{x \rightarrow+\infty} \int_{0}^{1}\left(r_{X}(t)-x\right)^{+} d t=0,
$$

where we have used the non-increasingness of the function $x \mapsto \int_{0}^{1}\left(r_{X}(t)-x\right)^{+} d t$ and the Lebesgue convergence theorem. For $y=-1$, we have

$$
\phi^{*}(-1)=\sup _{x \in \mathbb{R}}\left(-x-\int_{0}^{1}\left(r_{X}(t)-x\right)^{+} d t\right)=-\lim _{x \rightarrow-\infty} \int_{0}^{1} \max \left(r_{X}(t), x\right) d t=-\int_{0}^{1} r_{X}(t) d t .
$$

By analogous computations, we obtain that $\phi^{*}(y)=+\infty$ for $y>0$, as well as $\phi^{*}(y)=+\infty$ for $y<-1$.

Finally, let us consider the case where $y \in(-1,0)$.

The function $f$ defined by $f(x):=x y-\phi(x)$ is concave (the function $\phi$ being convex). Noticing that $f(x)=x y-\int_{x}^{+\infty}\left(1-G_{X}(u)\right) d u$, we see that the right-hand and left-hand derivatives of $f$ at $x$ are given by $f_{+}^{\prime}(x)=y+\left(1-G_{X}(x+)\right)$ and $f_{-}^{\prime}(x)=y+\left(1-G_{X}(x-)\right)$. A point $x$ is a maximum point for the function $f$ if $\left\{\begin{array}{l}f_{+}^{\prime}(x) \leq 0 \\ f_{-}^{\prime}(x) \geq 0\end{array}\right.$ which is equivalent to $\left\{\begin{array}{l}G_{X}(x+) \geq y+1 \\ G_{X}(x-) \leq y+1\end{array}\right.$ which, in turn, is equivalent to $x$ being a $(y+1)$-quantile of $X$. So, by taking $x=r_{X}(y+1)$, we have

$$
\phi^{*}(y)=y r_{X}(y+1)-\int_{0}^{1}\left(r_{X}(t)-r_{X}(y+1)\right)^{+} d t=-\int_{y+1}^{1} r_{X}(t) d t
$$

which concludes the proof.

Proof of Proposition 3.5 The implication (ii) $\Rightarrow(\mathrm{i})$ is obvious by taking $g(t):=\mathbb{I}_{[y, 1]}(t)$ which is non-negative, non-decreasing and integrable.

Let us now turn to the converse implication. Suppose that (i) holds true. The assertion (ii) is true for any function $g$ of the form $g(t):=\mathbb{I}_{[y, 1]}(t)$. 
Let now $g$ be a non-negative, non-decreasing step function. Then $g$ can be written as follows: $g(t)=\sum_{i=1}^{k} a_{i} \mathbb{I}_{[b i, 1]}$ where $a_{i} \geq 0$ and $0=b_{1}<b_{2} \cdots<b_{k}<1$. Thus, we have

$$
\int_{0}^{1} g(t) r_{X}(t) d t=\sum_{i=1}^{k} a_{i} \int_{b_{i}}^{1} r_{X}(t) d t \geq \sum_{i=1}^{k} a_{i} \int_{b_{i}}^{1} r_{Y}(t) d t=\int_{0}^{1} g(t) r_{Y}(t) d t .
$$

Let now $g$ be a non-negative, non-decreasing function. Then $g$ can be approximated from below by a sequence $\left(g_{n}\right)$ of non-negative, non-decreasing step functions. Due to the previous obser-

vation, we then have $\int_{0}^{1} g_{n}(t) r_{X}(t) d t \geq \int_{0}^{1} g_{n}(t) r_{Y}(t) d t$. The function $g$ being integrable and the functions $r_{X}$ and $r_{Y}$ being bounded (since $X$ and $Y$ are in $\chi$ ), we can apply the Lebesgue convergence theorem to pass to the limit in the previous inequality which concludes the proof.

\section{Appendix: The generalized Hardy-Littlewood's inequal- ities}

We give the proof of proposition 4.1.

Let us first prove the upper bound part in proposition 4.1. Before we proceed, we need the following two lemmas:

Lemma C.1 Let $\mu$ be a capacity on $(\Omega, \mathcal{F})$ which is continuous from below. Let $\left(X_{n}\right)$ be a nondecreasing sequence of non-negative measurable functions and let $X$ denote the limit function. Then the sequence of distribution functions (with respect to $\mu$ ) of $X_{n}$ converges to the distribution function (with respect to $\mu$ ) of $X$ i.e.

$$
\lim _{n \rightarrow \infty} G_{X_{n}}(x)=G_{X}(x), \forall x \in \overline{\mathbb{R}}_{+} .
$$

Proof: The proof of this lemma is contained in the proof of theorem 8.1 in Denneberg (1994) and is omitted.

Remark C.1 We note that lemma C.1 remains valid even when the non-negativity assumption on the functions of the sequence $\left(X_{n}\right)$ is relaxed. 
Lemma C.2 Let $\mu$ be a capacity on $(\Omega, \mathcal{F})$ which is continuous from below. Let $\left(X_{n}\right)$ be a nondecreasing sequence of non-negative measurable functions and let $X$ be a non-negative measurable function such that $X_{n} \uparrow X$. Then we have the following convergence:

$$
r_{X_{n}}(t) \uparrow r_{X}(t) \text { for almost every } t
$$

where $r_{X_{n}}$ and $r_{X}$ stand for (versions of) the quantile functions (with respect to $\mu$ ) of $X_{n}$ and $X$ respectively.

Proof: To prove the result we will use the lower quantile function $r_{X_{n}}^{l}$ of $X_{n}$ defined by:

$$
r_{X_{n}}^{l}(t):=\sup \left\{x \in \mathbb{R}: G_{X_{n}}(x)<t\right\}, \text { for } t \in(0,1) .
$$

The sequence $\left(X_{n}\right)$ being non-negative, non-decreasing, we have that the sequence $\left(r_{X_{n}}^{l}\right)$ is non-negative, non-decreasing and we denote by $r$ its limit function i.e. $r(t):=\lim _{n} r_{X_{n}}^{l}(t)=$ $\sup _{n} r_{X_{n}}^{l}(t), \forall t \in(0,1)$. We will show that $\forall t \in(0,1), r(t)=r_{X}^{l}(t)$ where $r_{X}^{l}(t):=\sup \{x \in \mathbb{R}:$ $\left.G_{X}(x)<t\right\}$ is the lower quantile function of $X$ (with respect to $\mu$ ). The conclusion of the lemma will follow as $r_{X}^{l}=r_{X}$ almost everywhere and $r_{X_{n}}^{l}=r_{X_{n}}$ almost everywhere.

Now, $G_{X_{n}} \geq G_{X} \forall n$, which implies that $r_{X_{n}}^{l}(t) \leq r_{X}^{l}(t), \forall t \in(0,1), \forall n$. By passing to the limit, we obtain $r(t) \leq r_{X}^{l}(t), \forall t \in(0,1)$.

We turn to the proof of the converse inequality, namely $r(t) \geq r_{X}^{l}(t), \forall t \in(0,1)$. Fix $t \in(0,1)$ and let $x \in \mathbb{R}$ be such that $G_{X}(x)<t$. By lemma C.1, we know that $G_{X_{n}}(x) \downarrow G_{X}(x)$. Hence, there exists $n_{0}=n_{0}(t, x)$ such that for all $n \geq n_{0}, G_{X_{n}}(x)<t$. Therefore, for all $n \geq n_{0}, x \in$ $\left\{y \in \mathbb{R}: G_{X_{n}}(y)<t\right\}$ which implies that $r_{X_{n}}^{l}(t):=\sup \left\{y \in \mathbb{R}: G_{X_{n}}(y)<t\right\} \geq x, \forall n \geq n_{0}$. By passing to the limit, we obtain that $r(t) \geq x$ which gives the desired inequality and concludes the proof.

Now, we are ready to prove proposition 4.1.

Proof of proposition 4.1: We will prove the first part of the proposition which concerns the upper bound. The lower bound is proved similarly.

Let $\mu$ be a continuous from below, concave capacity. The inequality is satisfied by $X$ and $Y$ of the form $X=\mathbb{I}_{A}, Y=\mathbb{I}_{B}$, where $A, B \in \mathcal{F}$ (even without the assumption of continuity from below and concavity of $\mu$ ). Indeed,

$$
\mathbb{E}_{\mu}\left(\mathbb{I}_{A} \mathbb{I}_{B}\right)=\mu(A \cap B) \leq \mu(A) \wedge \mu(B)=\int_{0}^{1} r_{\mathbb{I}_{A}}(t) r_{\mathbb{I}_{B}}(t) d t
$$


Similarly to the classical case we have that $r_{\mathbb{I}_{A}}=\mathbb{I}_{(1-\mu(A), 1]}$ a.e.; thus, the last equality in (C.1) is due to the following computation:

$$
\int_{0}^{1} r_{\mathbb{I}_{A}}(t) r_{\mathbb{I}_{B}}(t) d t=\int_{0}^{1} \mathbb{I}_{(1-\mu(A), 1]}(t) \mathbb{I}_{(1-\mu(B), 1]}(t) d t=1-\max \{1-\mu(A), 1-\mu(B)\}=\mu(A) \wedge \mu(B) .
$$

We next prove the desired inequality for non-negative step functions. Let $X$ and $Y$ be two nonnegative step functions. Then the function $X$ has the following representation $X=\sum_{i=1}^{n} x_{i} \mathbb{I}_{A_{i}}$, with $x_{i} \geq 0$ and $A_{i} \in \mathcal{F}$. Without loss of generality, we can suppose that the numbers $x_{i}$ are ranged in a descending order (i.e. $x_{1} \geq x_{2} \geq \cdots \geq x_{n} \geq 0$ ) and that the sets $A_{i}$ are disjoint. Thus, the function $X$ can be rewritten in the following manner: $X=\sum_{i=1}^{n} \tilde{x}_{i} \mathbb{I}_{\tilde{A}_{i}}$, where $\tilde{x}_{i}:=x_{i}-x_{i+1} \geq 0, x_{n+1}:=0$ and $\tilde{A}_{i}:=\cup_{k=1}^{i} A_{k}$. This representation proves to be very useful, as will be seen later on in the proof, because the functions $\tilde{x}_{i} \mathbb{I}_{\tilde{A}_{i}}$ and $\tilde{x}_{j} \mathbb{I}_{\tilde{A}_{j}}$ are a couple of comonotonic functions. In the same manner, the function $Y$ has the following representation: $Y=\sum_{j=1}^{m} \tilde{y}_{j} \mathbb{I}_{\tilde{B}_{j}}$ where $\tilde{y}_{j} \geq 0$ and $\tilde{B}_{j} \subset \tilde{B}_{j+1}$.

Thanks to the subadditivity of the Choquet integral with respect to a concave capacity and to the positive homogeneity of the Choquet integral, we have

$$
\mathbb{E}_{\mu}(X Y) \leq \sum_{i=1}^{n} \sum_{j=1}^{m} \tilde{x}_{i} \tilde{y}_{j} \mu\left(\tilde{A}_{i} \cap \tilde{B}_{j}\right)
$$

On the other hand, we see that $r_{X}=\sum_{i=1}^{n} r_{X_{i}}$ a.e. where we have set $X_{i}:=\tilde{x}_{i} \mathbb{I}_{\tilde{A}_{i}}$ and where $r_{X_{i}}$ designates a quantile function of $X_{i}$. Indeed, as mentioned above, the functions in the $\operatorname{sum} \sum_{i=1}^{n} \tilde{x}_{i} \mathbb{I}_{\tilde{A}_{i}}$ are pairwise comonotonic, and therefore, the functions $\sum_{i=1}^{k-1} \tilde{x}_{i} \mathbb{I}_{\tilde{A}_{i}}$ and $\tilde{x}_{k} \mathbb{I}_{\tilde{A}_{k}}$ are comonotonic which allows us to obtain the desired property by induction. By the same argument, $r_{Y}=\sum_{j=1}^{m} r_{Y_{j}}$ a.e. where $Y_{j}:=\tilde{y}_{j} \mathbb{I}_{\tilde{B}_{j}}$ and $r_{Y_{j}}$ designates a quantile function of $Y_{j}$. So,

$$
\int_{0}^{1} r_{X}(t) r_{Y}(t) d t=\sum_{i=1}^{n} \sum_{j=1}^{m} \tilde{x}_{i} \tilde{y}_{j} \int_{0}^{1} r_{\mathbb{I}_{\tilde{A}_{i}}}(t) r_{\mathbb{I}_{\tilde{B}_{j}}}(t) d t
$$

where the non-negativity of $\tilde{x}_{i}$ and $\tilde{y}_{j}$ and lemma A.1 have been used.

The proof for non-negative step functions is therefore finished as the first part of the proof about indicator functions implies that $\mu\left(\tilde{A}_{i} \cap \tilde{B}_{j}\right) \leq \int_{0}^{1} r_{\mathbb{I}_{\tilde{A}_{i}}}(t) r_{\mathbb{I}_{\tilde{B}_{j}}}(t) d t$.

Now, let $X$ and $Y$ be two measurable non-negative functions. Let $\left(X_{n}\right)$ be a sequence of nonnegative step functions such that $X_{n} \uparrow X$ and let $\left(Y_{n}\right)$ be a sequence of non-negative step functions such that $Y_{n} \uparrow Y$. This implies that $0 \leq X_{n} Y_{n} \uparrow X Y$ and we use the monotone convergence theorem (theorem A.1) to obtain $\lim _{n \rightarrow \infty} \mathbb{E}_{\mu}\left(X_{n} Y_{n}\right)=\mathbb{E}_{\mu}(X Y)$. On the other 
hand, by using lemma C.2, we obtain $0 \leq r_{X_{n}}(t) \uparrow r_{X}(t)$ for almost every $t$ and $0 \leq r_{Y_{n}}(t) \uparrow$ $r_{Y}(t)$ for almost every $t$; we thus conclude that $0 \leq r_{X_{n}}(t) r_{Y_{n}}(t) \uparrow r_{X}(t) r_{Y}(t)$ for almost every $t$. By applying the monotone convergence theorem for Lebesgue integrals to the sequence $\left(r_{X_{n}}(.) r_{Y_{n}}().\right)$, we have $\lim _{n \rightarrow \infty} \int_{0}^{1} r_{X_{n}}(t) r_{Y_{n}}(t) d t=\int_{0}^{1} r_{X}(t) r_{Y}(t) d t$ which concludes the proof.

\section{References}

Artzner, P., F. Delbaen, J. M. Eber, and D. Heath (1999): Coherent measures of risk, Mathematical finance 9(3), 203-228.

Bion-Nadal, J. (2009): Bid-ask dynamic pricing in financial markets with transaction costs and liquidity risk, Journal of mathematical economics 45, 738-750.

Carlier, G., and R. A. Dana (2005): Rearrangement inequalities in non-convex insurance models, Journal of mathematical economics 41, 483-503.

Carlier, G., and R. A. Dana (2006): Law invariant concave utility functions and optimization problems with monotonicity and comonotonicity constraints, Statistics and Decisions 24, 127152.

Chateauneuf, A., Kast, R. and Lapied, A. (1996), Choquet pricing for financial markets with frictions, Mathematical finance 6, 323-330.

Chateauneuf, A., R. A. Dana, and J. M. Tallon (2000): Optimal risk-sharing rules and equilibria with Choquet-expected-utility, Journal of mathematical economics 34, 191-214.

Dana, R. A. (2004): Market behaviour when preferences are generated by second-order stochastic dominance, Journal of mathematical economics 40, 619-639.

Dana, R. A. (2005): A representation result for concave Schur concave functions, Mathematical finance 15, 613-634.

Dana, R. A., and I. Meilijson (2003): Modelling agents' preferences in complete markets by second-order stochastic dominance, Cahier du Ceremade 0333. 
Denneberg, D. (1990): Premium calculation: why standard deviation should be replaced by absolute deviation, ASTIN Bulletin 20, 181-190.

Denneberg, D. (1994): Non-additive measure and integral, Kluwer Academic Publishers, Dordrecht.

Denuit, M., J. Dhaene, M. J. Goovaerts, R. Kaas, and R. Laeven (2006): Risk measurement with the equivalent utility principles, Statistics and Decisions 24, 1-25.

Dhaene, J., S. Vanduffel, M. J. Goovaerts, R. Kaas, Q. Tang, and D. Vyncke (2006): Risk measures and comonotonicity: a review, Stochastic models 22, 573-606.

Dybvig, P. (1987): Distributional analysis of portfolio choice, Journal of Business 61(3), 369-393,.

Ekeland, I., A. Galichon, and M. Henry (2009): Comonotonic measures of multivariate risks, Cahiers de l'Ecole polytechnique, Cahier 2009-25.

Föllmer, H., and A. Schied (2004): Stochastic finance. An introduction in discrete time, De Gruyter Studies in Mathematics, 2nd edition.

Grigorova, M. (2010): Choquet expected utility and related problems via quantile functions with respect to a capacity, working paper.

Grigorova, M. (2010): Stochastic orderings with respect to a capacity and risk measures, working paper.

Jouini, E., and H. Kallal (2001): Efficient trading strategies in the presence of market frictions, Review of Financial Studies 14(2), 343-369.

Kaas, R., M. J. Goovaerts, J. Dhaene, and M. Denuit (2001): Modern Actuarial Risk Theory, Kluwer Academic Publishers, Dordrecht.

Klöppel, S., and M. Schweizer (2007): Dynamic indifference valuation via convex risk measures, Mathematical finance 17, 599-627.

Müller, A., and D. Stoyan (2002): Comparison Methods for Stochastic Models and Risks, Wiley Series in Probability and Statistics, Wiley. 
Ogryczak, W., and A. Ruszczynski (2001): Dual stochastic dominance and related mean-risk models, Research report, Rutgers center for operations research, Rutgers University.

Quiggin, J. (1982): A theory of anticipated utility, Journal of Economic Behavior and Organisation 3, 323-343.

Rockafellar, R. T. (1972): Convex Analysis, Princeton University Press.

Shaked, M., and G. Shanthikumar (2006): Stochastic Orders, Springer Series in Statistics, Springer.

Wang, S., V. Young, and H. Panjer (1997): Axiomatic characterization of insurance prices, Insurance: Mathematics and Economics 21, 173-183.

Wang, S., and V. Young (1998): Ordering risks: Expected utility theory versus Yaari's dual theory of risk, Insurance: Mathematics and Economics 22, 145-161.

Wang, Z., and J. A. Yan (2007): A selective overview of applications of Choquet integrals, Advanced Lectures in Mathematics, Higher Educational Press and International Press, 484514.

Yaari, M. (1997): The dual theory of choice under risk, Econometrica 55, 95-115.

Young, V. (2004): Premium calculation principles, Encyclopedia of Actuarial Science, John Wiley, New York. 